Perspectiva de Familia (Perspect. fam.) ISSN 2415-5187, año 2018, vol. 3, pp. 9-24

\title{
Evidencias preliminares de validez de contenido, validez convergente y confiabilidad del Îtem Único de Satisfacción con la vida familiar (IUSWFL) en adolescentes limeños
}

\author{
Preliminary Evidence of Content Validity, \\ Convergent Validity and Reliability of the Single Item of Satisfaction \\ with Family Life (IUSWFL) in Adolescents from Lima \\ Tomás Caycho-Rodríguez', Marilyn J. Cuellar-Llata ${ }^{1}$, \\ Renzo Felipe Carranza Esteban², Isabel Cabrera-Orosco³, \\ Valeria G. Franco-Reynoso', Jennifer D. Casariego-Oviedo', \\ Melanie Mendives-Delgado', Lisseth Rojas-Ayala', \\ Fiorella A. Torrejón-Pinedo ${ }^{1}$ \\ 'Universidad Privada del Norte, Lima, Perú \\ ${ }^{2}$ Universidad Peruana Unión, Tarapoto, Perú \\ 3Universidad Continental, Lima, Perú
}

Recibido: 03-06-18

Aceptado: 10-08-18

\section{Resumen}

El estudio examinó las evidencias de validez de contenido, validez convergente y confiabilidad de una medida de ítem único de satisfacción con la vida familiar (IUSWFL). Los participantes fueron 509 adolescentes, hombres y mujeres, entre 10 y 17 años $(M=13.31$ años; $D E=1.6)$ de la ciudad de Lima. Se utilizó la Satisfaction with Family Life Scale (SWFL), el cuestionario APGAR Familiar y el IUSWFL. Para las evidencias de validez de contenido se utilizó el método de jueces expertos cuantificando sus valoraciones con la V de Aiken. La validez convergente se realizó a partir de un análisis comparativo con el ANOVA de una vía, así como un análisis de correlación con el coeficiente de Pearson. La confiabilidad fue analizada con el coeficiente alfa con el cálculo de sus intervalos de confianza. Los resultados sugieren evidencias iniciales de validez de contenido $(V>.70)$ y validez convergente. Se observan promedios ascendentes en funcionalidad familiar y satisfacción con la vida familiar a medida que los grupos, originados en función de las alternativas de 
Tomás Caycho, Marilyn J. Cuellar, Renzo Carranza, Isabel Cabrera, Valeria Franco, Jennifer Casariego, Melanie Mendives, Lisseth Rojas \& Fiorella A. Torrejón

respuesta del IUSWFL, expresen un mayor grado de acuerdo. El IUSWFL se asoció positivamente con la SWFL. Los valores de los coeficientes de confiabilidad fueron adecuados. Se concluye que el IUSWFL cuenta con evidencias de validez de contenido, validez convergente y confiabilidad para ser utilizado como una medida breve de la SWFL.

Palabras clave: confiabilidad, medida de ítem único, satisfacción con la vida familiar, validez convergente, validez de contenido.

\begin{abstract}
The study examined the evidence of content validity, convergent validity and reliability of a single item measure of satisfaction with family life (IUSWFL). The participants were 509 adolescents, men and women, between 10 and 17 years old $(\mathrm{M}=13.31, \mathrm{SD}=1.6)$ from the city of Lima. The Satisfaction with Family Life Scale (SWFL), the Family APGAR Questionnaire and the IUSWFL were used. For evidence of content validity, the method of expert judges was used quantifying their valuations with the $\mathrm{V}$ of Aiken. The convergent validity was made from a comparative analysis with one-way ANOVA, as well as a correlation analysis with the Pearson coefficient. Reliability was analyzed with the alpha coefficient with the calculation of their confidence intervals. The results suggest initial evidence of content validity $(\mathrm{V}>.7 \mathrm{O})$ and convergent. There are ascending averages in family functionality and satisfaction with family life as the groups, originating in response to the IUSWFL alternatives, express a greater degree of agreement. The IUSWFL was positively associated with the SWFL. The values of reliability coefficients were adequate. It is concluded that the IUSWFL has evidences of content validity, convergent validity and reliability to be used as a brief measure of the SWFL
\end{abstract}

Keywords: reliability, single item measurement, satisfaction with family life, convergent validity, content validity.

La literatura científica señala que la satisfacción con la vida (SWL, por sus siglas en inglés) está altamente asociada con la salud y el bienestar subjetivo (Huebner, Suldo, Smith \& McKnight, 2004), y es muy importante en el adecuado funcionamiento psicológico, educativo, social y físico de los adolescentes en edad escolar (Gilman \& Huebner, 2003; Huebner, 2004; Suldo, Huebner, Friedrich \& Gilman, 2009). De esta manera, la presencia de SWL en adolescentes está asociada con una ausencia de psicopatología (Suldo \& Shaffer, 2008), mayores aspiraciones académicas y actitudes más favorables hacia los docentes y la escuela (Lewis, Huebner, Malone \& Valois, 2011; Proctor, Linley \& Maltby, 2010), y mejores hábitos saludables (González- 
Evidencias preliminares de validez de contenido, validez convergente y confiabilidad del Item Único de Satisfacción con la vida familiar (IUSWFL) en adolescentes limeños

Serrano, Huéscar \& Moreno-Murcia, 2013). De igual manera, los adolescentes con niveles adecuados de SWL presentan un mejor rendimiento académico percibido (Shek \& Li, 2016), pueden afrontar mejor las consecuencias del desempleo familiar (Frasquilho, Matos, Neville, Gaspar \& Almeida, 2016), no presentan comportamientos riesgosos (Çakar, Tagay \& Karataș, 2015) ni son víctimas de bullying (Totan, Özer \& Özmen, 2017).

Si bien la SWL es definida como la evaluación que un individuo realiza de su vida en general (Diener \& Ryan, 2009), se enfoca también en una evaluación global de determinados dominios específicos de la vida (Easterlin, 2006; Diener \& Ryan, 2009), como el trabajo, la familia, los amigos, la actividad física, las finanzas y el ocio, entre otros (Huebner, Suldo \& Gilman, 2006; Joo \& Grable, 2004; Plagnol, 2011; Tsou \& Liu, 2001; Van Praag \& Ferrer-i-Carbonell, 2004). De los factores mencionados, el dominio familiar no ha recibido mucha atención en la literatura científica en comparación con otros dominios de la satisfacción con la vida (Botha, Booysen \& Wouters, 2017); además de que los pocos estudios que han centrado su interés en la satisfacción con la vida familiar lo han hecho de manera tangencial (Agate, Zabriskie, Agate \& Poff, 2009; Yamamura, 2014; Moss \& Willoughby, 2016). Todo esto, a pesar de que la familia es un componente importante de la sociedad (Zabriskie \& Ward, 2013) e influye en el desarrollo psicológico y social de los adolescentes (Alesina \& Giuliano, 2013; Conger, Conger \& Martin, 2010), quienes consideran a la familia como una fuente importante de apoyo y protección (Arnett, 20o8; Barboza-Palomino et al., 2017), por encima incluso de lo que la escuela o la religión pueden ofrecerles (Serrano \& Flores, 2004).

Estudios con adolescentes en edad escolar reportan que la satisfacción con la vida familiar (SWFL, por sus siglas en inglés) favorece la expresión de afectos positivos y la satisfacción con otros dominios de la vida, como los amigos, el trabajo, la salud y la escuela (Luna, Laca \& Mejía, 2011). Además, la SWFL promueve la prosocialidad y la autoestima, mientras que la insatisfacción familiar es comúnmente acompañada de problemas sociales y emocionales (Gilman, 20o1; Valois, Zullig, Huebner \& Drane, 2001).

Lo anterior genera la necesidad de disponer de una medida confiable, válida y útil de la SWFL (Tasdelen-Karçkay, 2016). Si bien se considera que los instrumentos de medida conformados por ítems múltiples son superiores a las medidas breves y que, específicamente en psicología, se ha observado un impulso psicométrico hacia el desarrollo de escalas largas, en la actualidad, muchas situaciones requieren el uso de medidas cortas por razones pragmáticas (Postmes, Haslam \& Jans, 2013). Así, los períodos de atención más cortos y el interés por recopilar información de una gran cantidad de personas en un menor tiempo y a través de medios como 
Tomás Caycho, Marilyn J. Cuellar, Renzo Carranza, Isabel Cabrera, Valeria Franco, Jennifer Casariego, Melanie Mendives, Lisseth Rojas \& Fiorella A. Torrejón

teléfonos móviles o redes sociales generan la necesidad de medidas más cortas (Alam, Khusro, Rauf \& Zaman, 2014; Konrath, Meier \& Bushman, 2018).

La literatura sugiere que las medidas cortas pueden ser tan válidas y confiables como los instrumentos largos (Burisch, 1984). Resultados empíricos apoyan la idea de que las escalas cortas pueden alcanzar niveles aceptables de confiabilidad si evalúan constructos homogéneos y claramente definidos (Loo \& Kelts, 1998).

La forma más corta que un instrumento puede tomar es una medida de ítem único (MIU) que trae consigo escepticismo y preocupación metodológica (Postmes, Haslam \& Jans, 2013). A pesar de las limitaciones propias de la MIU (Fuch \& Diamantopoulus, 2009; Loo, 2002), en la actualidad existen desarrollos metodológicos para estimar su confiabilidad y las evidencias de validez, dependiendo de la naturaleza del constructo operacionalizado (Wanous, Reichers \& Hudy, 1997; Wanous \& Hudy, 20o1). Como resultado de tales desarrollos, en el Perú, un número creciente de ítems únicos se ha desarrollado y está disponible para medir constructos tales como la ansiedad ante los exámenes (Dominguez-Lara, 2017) y el burnout (Fernández-Arata, Merino-Soto \& Dominguez-Lara, 2017).

A pesar del amplio escepticismo hacia las escalas cortas, y las MIU en particular, estas presentan beneficios, pues permiten una reducción de la fatiga y el aburrimiento asociados a contestar pruebas largas y con ítems repetitivos (Van Hoof, Geurts, Kompier \& Taris, 2007). Además, las MIU son útiles en estudios empíricos para la formación de grupos a partir de sus respuestas y como medida de control (Boyd, Gove \& Hitt, 2005). Además, las MIU favorecen las oportunidades de investigación, ya que brindan más espacio en las encuestas para la inclusión de otros constructos relevantes (Onwezen, Reinders, Verain \& Snoek, 2019).

En este sentido, en la presente investigación se propone una MIU para medir la SWFL en adolescentes (IUSWFL, por sus siglas en inglés) que corresponde al ítem 3 de la versión peruana de la Satisfaction with Family Life Scale (Caycho-Rodríguez et al., 2018), cuyo contenido es "Estoy satisfecho(a) con mi vida familiar". El ítem fue elegido en función de su redacción directamente relacionada con el constructo evaluado (en este caso, satisfacción con la vida familiar), en el que un alto puntaje evidencia una mayor presencia de la satisfacción con la vida familiar. Asimismo, el ítem 3 presentó la mayor carga factorial en el estudio de validación peruano (Caycho-Rodríguez et al., 2018), lo que brinda evidencia de que el ítem se encuentra conceptualmente más cerca del constructo SWFL (West, Dyrbye, Satele, Sloan \& Shanafelt, 2012). Finalmente, debido a la brevedad del ítem, conformado por 6 palabras, se espera un aumento de la calidad de las respuestas de los encuestados, al ser más directas y menos sesgadas (Lenzner, Kaczmirek \& Lenzner, 2010; Sloan et al., 2002). 
Evidencias preliminares de validez de contenido, validez convergente y confiabilidad del Item Único de Satisfacción con la vida familiar (IUSWFL) en adolescentes limeños

Considerando lo anterior, el objetivo del presente estudio fue analizar las evidencias iniciales de validez de contenido, validez convergente y confiabilidad del IUSWFL en adolescentes peruanos en edad escolar.

\section{Método}

\section{Participantes}

Un total de 509 adolescentes entre 10 y 17 años $(M=13.31$ años; $D E=1.6)$ de la ciudad de Lima participaron en el estudio. El grupo de mujeres estaba compuesto por 250 adolescentes de una edad promedio de 13.27 años $(\mathrm{DE}=1.58)$, mientras que el grupo de hombres estaba constituido por 258 adolescentes de una edad promedio de 13.36 años $(\mathrm{DE}=1.62)$. Entre estos niños, 317 vivían con ambos padres $(62.3 \%)$, 109 vivían solo con la madre ( $21.4 \%), 56$ no vivían con sus padres pero sí con otros familiares (11 \%), 26 vivían solo con el padre (5.1\%) y 1 vivía solo (o.2 \%). En cuanto a la edad, no se evidencian diferencias significativas entre hombres y mujeres $\left(t_{(506)}=.678, p>.05 ; d=.06 ; I_{95} \%:-.12, .23\right)$.

En el análisis de validez de contenido, participaron to jueces expertos, todos ellos psicólogos profesionales (siete varones y tres mujeres), cuyas áreas de acción eran la psicología clínica ( $88.7 \%$ ) y la educativa (11.3\%), y con un promedio de 15.8 años de experiencia profesional $(\mathrm{DE}=8.24)$. Todos contaban con los grados académicos de magíster o doctor.

\section{Instrumentos}

Ítem Único de Satisfacción con la Vida Familiar (IUSWFL). Se trata de una medida unidimensional de la satisfacción con la vida familiar, que corresponde al ítem 3 de la Satisfaction with Family Life Scale (SWFLS; Zabriskie \& McCormick, 2003), adaptada a adolescentes peruanos (Caycho-Rodríguez et al., 2018). Su contenido es "Estoy satisfecho(a) con mi vida familiar", que tiene 7 alternativas de respuesta (desde "Totalmente en desacuerdo" hasta "Totalmente de acuerdo").

Cuestionario APGAR Familiar (Smilkstein, 1978). Mide cinco áreas del funcionamiento familiar (adaptabilidad, cooperación, desarrollo, afectividad y capacidad resolutiva). Las cuatro opciones de respuesta tipo Likert (desde "Nunca" hasta "Siempre") brindan un puntaje que varía de o a 20 , que indica un mejor funcionamiento familiar. La versión peruana ha demostrado evidencia de fiabilidad y validez de constructo en adolescentes de la ciudad de Lima (Castilla, Caycho, Ventura, Palomino-Barboza \& De la Cruz, 2015). La consistencia interna, calculada mediante el coeficiente alfa de Cronbach, para el presente estudio fue o.82 (IC95\%: .79-.85). 
Tomás Caycho, Marilyn J. Cuellar, Renzo Carranza, Isabel Cabrera, Valeria Franco, Jennifer Casariego, Melanie Mendives, Lisseth Rojas \& Fiorella A. Torrejón

Satisfaction with Family Life Scale (SWFLS; Zabriskie \& McCormick, 2003). Mide el juicio cognitivo acerca de la propia vida familiar a partir del grado de acuerdo o desacuerdo con 5 ítems que tienen una escala tipo Likert de siete alternativas de respuesta (de $1=$ totalmente en desacuerdo 7 = totalmente de acuerdo). La SWFLS ha demostrado evidencias adecuadas de confiabilidad y validez para adolescentes peruanos (Caycho-Rodríguez et al., 2018). En el presente estudio, se omitió el ítem 3 para el cómputo del puntaje total. La consistencia interna, calculada mediante el coeficiente alfa de Cronbach, fue o.8o (IC $95 \%$ : .77-.83).

\section{Procedimiento}

El protocolo del estudio recibió el aval del comité de ética de la Universidad Privada del Norte. Primero, previa información de las características y el objetivo de la investigación, se solicitó y se recibió la autorización de las instituciones educativas de nivel secundario para la aplicación del IUSVF y los demás instrumentos de medición. Posteriormente, se coordinó con los docentes y se aplicaron los instrumentos de manera colectiva en las aulas de los estudiantes. Antes de esto, se comunicó a los estudiantes el objetivo del estudio y se obtuvo su participación voluntaria a través de la firma del consentimiento informado.

\section{Análisis de datos}

En primer lugar, la evidencia de validez de contenido se evaluó a partir del análisis de la claridad, la coherencia y la relevancia de los ítems del IUSWFL. Cada uno de estos criterios se calificó dentro de un rango de o (nada relevante/coherente/claro) a 3 (totalmente relevante/coherente/claro). Para la cuantificación del grado de claridad, coherencia y relevancia de los ítems del IUSWFL se utilizó el coeficiente $V$ de Aiken (Aiken, 1980) y sus intervalos de confianza al $95 \%$ (IC95\%), a partir de lo planteado por Penfield y Giacobbi (Penfield \& Giacobbi, 2004). La $V$ puede tomar valores que oscilan entre o y 1 , donde valores cercanos a 1 expresan un mayor grado de claridad, coherencia y relevancia del ítem. De igual forma, valores de $V$ mayores a 0.70 indican una valoración positiva del ítem a nivel muestral; mientras que, a nivel poblacional, valores del límite inferior del IC $95 \%$ mayores a 0.59 son considerados adecuados.

En segundo lugar, se calcularon estadísticos descriptivos (media, desviación estándar, asimetría y curtosis) para el IUSWFL. Posteriormente, las evidencias de validez convergente se analizaron desde una perspectiva asociativa. En primer lugar, se compararon los promedios de la funcionalidad familiar (medida con el APGAR) y de la satisfacción con la vida familiar (medida con la SWFLS sin el ítem 3) entre cada uno de los grupos conformados a partir de las siete opciones de respuesta del 
Evidencias preliminares de validez de contenido, validez convergente y confiabilidad del Item Único de Satisfacción con la vida familiar (IUSWFL) en adolescentes limeños

IUSWFL. Se utilizó para esto la prueba de ANOVA de una vía, mientras que el tamaño del efecto fue estimado a partir del coeficiente omega cuadrado $\left(\omega^{2}\right)$, donde valores de $0.04,0.25$ y 0.64 expresan un tamaño del efecto pequeño, moderado y fuerte (Caycho-Rodríguez, 2017a; Ferguson, 2009).

Asimismo, se calculó el coeficiente de correlación de Pearson $(r)$ entre la puntuación del IUSWFL y otras medidas de satisfacción con la vida familiar (SWFLS) y la funcionalidad familiar (APGAR). Se calculó el intervalo de confianza (IC) del coeficiente $r$ y el tamaño del efecto, a partir de un módulo MS Excel ${ }^{\circledR}$ ad hoc. Valores de $r \geq .20, r \geq .50$ y $r \geq .80$ expresan un tamaño del efecto pequeño, moderado y grande, respectivamente (Caycho-Rodríguez, 2017b). La magnitud de la correlación entre el IUSWFL y la ESVF ( $\sin$ el ítem 3 ) entre el grupo de varones y mujeres fue comparada con el estadístico $q$ (Cohen, 1992), donde $\mathrm{q} \leq .10, \mathrm{q}>.10, \mathrm{q}>.30$ y q $>$ .50 expresan una diferencia trivial, pequeña, moderada y grande, respectivamente (Ventura-León \& Caycho, 2017).

Se estimó la confiabilidad mediante el cálculo del coeficiente alfa de Cronbach para ítem único (Dominguez-Lara \& Merino-Soto, 2017; Wanous \& Hudy, 2001) con sus IC sobre la base del método Fisher, que no toma en cuenta el número de ítems (IC; Dominguez-Lara \& Merino-Soto, 2015). Se esperan magnitudes mayores a 0.80 para el coeficiente $\alpha$ (Merino, Navarro \& García, 2014). Asimismo, se compararon los coeficientes alfa del IUSVF entre hombres y mujeres utilizando el método de Feldt (Feldt, Woodruff \& Salih, 1987) a partir del programa AlphaTest (Lautenschlager \& Meade, 2008).

\section{Resultados}

\section{Evidencia de validez de contenido}

A nivel muestral $(V>.70)$ y poblacional $(L i>.59)$, el ítem recibió evaluaciones favorables acerca de su claridad, su coherencia y su relevancia, y obtuvo un valor $V=1.00$ (IC95\%: .92-1.0o) para todos estos criterios. Además, todos los valores del coeficiente $V$ fueron estadísticamente significativos.

\section{Análisis descriptivo de los ítems}

La Tabla 1 reporta el análisis descriptivo del IUSWFL en la muestra total y por sexo. Respecto a la muestra total, las respuestas predominantes en el IUSWFL fueron elevadas $(\mathrm{M}=5.39 ; \mathrm{DE}=1.68)$, donde el porcentaje de frecuencia fue "Totalmente de acuerdo" (33.8\%), "De acuerdo" (25.7\%), "Ligeramente de acuerdo" (12.6\%), "Ni de acuerdo ni en desacuerdo" (12.6\%), "Ligeramente en desacuerdo" (7.7\%), "En 
desacuerdo" (4.1\%) y “Totalmente en desacuerdo" (3.5\%). Una similar distribución de los porcentajes de respuesta se observa en la muestra de varones y mujeres. En relación con el sexo, existe una diferencia pequeña en el puntaje promedio del IUSWFL a favor de los varones $(d=.31$; IC95\%: .13-.48).

Tabla 1.

Estadísticos descriptivos del IUSVF y porcentaje de opciones de respuesta por sexo

\begin{tabular}{|ccccccccccccc|}
\hline & \multicolumn{4}{c}{ Estadísticos Descriptivos } & \multicolumn{1}{c|}{ Porcentaje de Opciones de Respuesta } \\
\cline { 2 - 12 } & M & DE & g1 & g2 & 1 & 2 & 3 & 4 & 5 & 6 & 7 \\
\hline Total & 5.39 & 1.68 & -0.95 & -0.01 & 3.5 & 4.1 & 7.7 & 12.6 & 12.6 & 25.7 & 33.8 \\
Varones & 5.64 & 1.51 & -1.11 & 0.45 & 1.2 & 4.3 & 5.8 & 9.7 & 14.3 & 26.7 & 38 \\
Mujeres & 5.13 & 1.81 & -0.76 & -0.44 & 6 & 4 & 9.6 & 15.6 & 10.8 & 24.4 & 29.6 \\
\hline
\end{tabular}

$\mathrm{M}=$ media; $\mathrm{DE}$ = desviación estándar; $\mathrm{g} 1$ = coeficiente de asimetría de Fisher; $\mathrm{g} 2$ = coeficiente de curtosis de Fisher; 1 = totalmente en desacuerdo; 2 = en desacuerdo; 3 = ligeramente en desacuerdo; 4 = ni de acuerdo ni en desacuerdo; 5 = ligeramente de acuerdo; 6 = de acuerdo; $7=$ totalmente de acuerdo.

El análisis de comparación de medias muestra diferencias estadísticamente significativas y un moderado tamaño del efecto entre los grupos (construidos a partir de cada tipo de respuesta del IUSWFL) en las variables funcionalidad familiar $(F=$ $\left.45.393 ; p=.000 ; \omega^{2}=.35\right)$ y satisfacción con la vida familiar $\left(F=94.279 ; p=.000 ; \omega^{2}\right.$ $=.53$ ). Se observan promedios ascendentes en funcionalidad familiar y satisfacción con la vida familiar a medida que los grupos expresan un mayor grado de acuerdo (ver Tabla 2). 
Evidencias preliminares de validez de contenido, validez convergente y confiabilidad del Item Único de Satisfacción con la vida familiar (IUSWFL) en adolescentes limeños

Tabla 2.

Evidencia de validez convergente

\begin{tabular}{|c|c|c|c|c|c|c|}
\hline & \multicolumn{3}{|c|}{ Análisis Descriptivo } & \multicolumn{3}{|c|}{ Análisis Comparativo } \\
\hline & IUSFV & M & DE & $F$ & $p$ & $\omega^{2}$ \\
\hline \multirow[t]{7}{*}{ Funcionalidad Familiar } & $\mathrm{TD}(1)$ & 6.28 & 5.61 & $45 \cdot 393$ & .000 & .35 \\
\hline & $\mathrm{D}(2)$ & 7.67 & 4.05 & & & \\
\hline & $\operatorname{LD}(3)$ & 9.26 & 4.06 & & & \\
\hline & $\mathrm{AD}(4)$ & 10.06 & 3.76 & & & \\
\hline & $\mathrm{LA}(5)$ & 11.72 & 3.77 & & & \\
\hline & $A(6)$ & 13.49 & 3.35 & & & \\
\hline & $\mathrm{TA}(7)$ & 15.24 & 3.04 & & & \\
\hline \multirow{7}{*}{$\begin{array}{l}\text { Satisfacción con la } \\
\text { Vida Familiar' }\end{array}$} & $\operatorname{TD}(1)$ & 9.61 & 5.01 & 94.279 & .000 & .53 \\
\hline & $\mathrm{D}(2)$ & 11.67 & 3.94 & & & \\
\hline & LD (3) & 14.08 & 4.75 & & & \\
\hline & $\mathrm{AD}(4)$ & 15.44 & 3.64 & & & \\
\hline & $\mathrm{LA}(5)$ & 18.77 & 4.41 & & & \\
\hline & $A(6)$ & 20.97 & 3.38 & & & \\
\hline & TA ( 7$)$ & 23.39 & 3.51 & & & \\
\hline
\end{tabular}

'Excluyendo el item 3; $\mathrm{M}=$ media; $\mathrm{DE}=$ desviación estándar; $\mathrm{TD}=$ totalmente en desacuerdo; $\mathrm{D}=$ en desacuerdo; $\mathrm{LD}=$ ligeramente en desacuerdo; $\mathrm{AD}=$ ni de acuerdo ni en desacuerdo; $\mathrm{LA}=$ ligeramente de acuerdo; $\mathrm{A}=$ de acuerdo; $\mathrm{TA}=$ totalmente de acuerdo.

Asimismo, la correlación entre el puntaje promedio del IUSWFL y la ESVF, sin el ítem 3, fue de 0.83 ( $p<0.001$; IC95\%: o.8o, o.92) para la muestra total; mientras que para varones y mujeres se observa un valor de $r$ de 0.83 ( $p<0.001$; IC95\%: 0.79, o.93) у 0.81 ( $p<0.001$; IC $95 \%$ : o.76, o.92), respectivamente. Todas las correlaciones presentan un tamaño del efecto fuerte (Caycho-Rodríguez, 2017). No se reportaron diferencias al comparar la magnitud de las correlaciones entre el IUSWFL y la ESVF entre varones y mujeres ( $q=0.06$; IC95\%: -0.03, o.15) (Ventura-León \& Caycho, 2017). Los resultados brindan evidencia favorable de validez convergente.

\section{Confiabilidad}

La confiabilidad del IUSWFL, estimada por el método de consistencia interna, fue para la muestra total $\alpha=0.81$ (IC95\%: 0.78, o.84). De igual manera, al analizar 
Tomás Caycho, Marilyn J. Cuellar, Renzo Carranza, Isabel Cabrera, Valeria Franco, Jennifer Casariego, Melanie Mendives, Lisseth Rojas \& Fiorella A. Torrejón

las muestras de varones y mujeres, se obtuvieron coeficientes alfa de 0.81 (IC $95 \%$ : 0.76, o.85) y 0.78 (IC95\%: 0.73, o.82), respectivamente. Todos los valores de alfa están por encima de lo recomendado para la investigación básica $(\alpha>$.70) (Merino, Navarro \& García, 2014). De forma adicional, los IC $95 \%$ de las diferencias entre los valores del coeficiente alfa de la muestra de varones y mujeres (IC95\%: -0.09-0.03) no son estadísticamente significativos, lo que indica una igualdad en la estimación del error del IUSVF entre ambos grupos.

\section{Discusión}

A pesar de la existencia de medidas de SWFL válidas y confiables, pero con gran cantidad de ítems, en la actualidad existe apoyo para el desarrollo de las MIU (Wanous et al., 1997; Wanous \& Hudy, 2001). Sin embargo, son pocos los estudios que han examinado las evidencias de validez de contenido, validez convergente y confiabilidad de las MIU, debido a la dificultad para examinar las propiedades psicométricas de estas medidas. En este sentido, el objetivo del estudio fue proporcionar evidencia psicométrica inicial de una MIU de la SWFL en adolescentes limeños.

Los resultados de este estudio proporcionan evidencia inicial que sugiere que el IUSWFL puede ser tan válido como las medidas más largas de SWFL y que se correlacionan muy fuertemente entre sí en muestras de adolescentes hombres y mujeres. En primer lugar, a pesar de ser solo un ítem, la evidencia de validez de contenido expresa que el IUSWFL es lo suficientemente relevante, coherente y claro para representar adecuadamente el constructo satisfacción con la vida familiar, tanto a nivel de muestra como de población. Esto es importante, ya que la omisión de este tipo de evidencia puede ser un error, debido a la diferencia en el significado y la interpretación que puede tener un constructo (en este caso, satisfacción con la vida familiar) como resultado de los diferentes contextos culturales.

De igual forma, el IUSWFL y la ESVF se correlacionaron fuertemente entre sí. Más importante aun, el IUSWFL se correlacionó con variables externas (funcionalidad familiar) en un grado similar. De igual manera, los resultados apoyan la hipótesis inicial en relación con la SWFL y la funcionalidad familiar, ya que las medias de ambas medidas muestran un incremento significativo mientras mayor sea la puntuación del IUSWFL. Asimismo, el análisis descriptivo previo reporta información valiosa acerca de la capacidad de discriminación del IUSWFL para identificar niveles elevados de SWFL. En la presente investigación, el 59.5 \% de los adolescentes respondieron con las dos opciones de mayor valor ("De acuerdo" y "Totalmente de acuerdo"). Este resultado indica la probabilidad de que aquellas personas que respondan alguna de estas opciones presenten una elevada SWFL. 
Evidencias preliminares de validez de contenido, validez convergente y confiabilidad del Item Único de Satisfacción con la vida familiar (IUSWFL) en adolescentes limeños

Respecto a la confiabilidad, la magnitud elevada del coeficiente alfa del IUSWFL expresa la presencia de un error de medición permisible. Al comparar los coeficientes alfa de las muestras de hombres y mujeres, indica que la estimación del error del IUSVF es similar en ambos grupos, lo que favorecería una medición más precisa.

El presente estudio tiene algunas limitaciones. En primer lugar, solo se aplicó a adolescentes de la ciudad de Lima, por lo que los resultados no pueden aplicarse a otras poblaciones de adolescentes. Sin embargo, los resultados favorables permiten esperar que el IUSWFL sea igualmente útil en poblaciones de otras regiones del Perú. En segundo lugar, no se examinó la sensibilidad y la especificidad, a pesar de que algunos autores sugieren que las MIU pueden ser menos sensibles cuando se usan con fines de investigación (Cheung \& Lucas, 2014). Así, estudios futuros acerca de la sensibilidad serán importantes para los investigadores que deseen utilizar el IUSWFL para evaluar la efectividad de las intervenciones o los programas que trabajen sobre la SWFL.

En resumen, el IUSWFL cuenta con evidencias iniciales de validez de contenido, validez convergente y confiabilidad. Este ítem puede ser útil cuando se requiera una medición global e integral de la SWFL de los adolescentes limeños o cuando las limitaciones de tiempo requieran una evaluación muy breve. Por consiguiente, el IUSWFL no pretende reemplazar otras medidas de la SWFL más largas y con más evidencias psicométricas. Si se desea una evaluación más detallada de la SWFL, ya sea con fines de diagnóstico o investigación, una medida con mayor cantidad de ítems puede seguir siendo la mejor opción de un psicólogo especialista en familia o un investigador.

\section{Referencias}

Agate, J. R., Zabriskie, R. B., Agate, S. T., \& Poff, R. (2009). Family leisure satisfaction and satisfaction with family life. Journal of Leisure Research, 41(2), 205223. doi: 10.108o/00222216.2009.11950166

Aiken, L. R. (1980). Content validity and reliability of single items or questionnaires. Educational and Psychological Measurement 40(4), 955-959. doi: 10.1177/001316448004000419

Alam, I., Khusro, S., Rauf, A., \& Zaman, Q. (2014). Conducting surveys and data collection: From traditional to mobile and SMS-based surveys. Pakistan Journal of Statistics and Operation Research, 10(2), 169-187. doi: 10.18187/pjsor. vioiz. 758

Alesina, A., \& Giuliano, P. (2013). Family ties. NBER Working Paper No. 18966. Massachusetts: National Bureau of Economic Research. 
Tomás Caycho, Marilyn J. Cuellar, Renzo Carranza, Isabel Cabrera, Valeria Franco, Jennifer Casariego, Melanie Mendives, Lisseth Rojas \& Fiorella A. Torrejón

Arnett, J. J. (2008). Adolescencia y adultez emergente. Un enfoque cultural (3. $\left.{ }^{\mathrm{a}} \mathrm{ed}.\right)$. México: Pearson Educación.

Barboza-Palomino, M., Moori, I., Zárate, S., López, A., Muñoz, K., \& Ramos, S. (2017). Influencia de la dinámica familiar percibida en el proyecto de vida en escolares de una institución educativa de Lima. Psicología Escolar e Educacional, 21(2), 157-166. doi: 10.1590/2175-3539201702121094

Botha, F., Booysen, F., \& Wouters, E. (2017). Satisfaction with family life in South Africa: The role of socioeconomic status. Journal of Happiness Studies, 19(8), 2339-2372. doi: 10.1007/s10902-017-9929-Z

Boyd, B. K., Gove, S., \& Hitt, M. A. (2005). Construct measurement in strategic management research: illusion or reality? Strategic Management Journal, 26(3), 239-257. doi: 10.1002/smj.444

Burisch, M. (1984). You don't always get what you pay for: Measuring depression with short and simple versus long and sophisticated scales. Journal of Research in Personality, 18(1), 81-98. doi: 10.1016/oo92-6566(84)90040-o

Çakar, F. F., Tagay, Ö. O., \& Karataş, Z. Z. (2015). Adolescents' life satisfaction: risky behaviors and hopelessness. International Journal on New Trends in Education \& Their Implications, 6(1), 55-62.

Castilla, H., Caycho, T., Ventura, J. L., Barboza, M., \& De la Cruz, M. (2015). Análisis factorial confirmatorio de la escala de percepción del funcionamiento familiar de Smilkstein en adolescentes peruanos. Salud E Sociedad, 6(2), 140-153. doi: 10.22199/So7187475.2015.0002.00003

Caycho-Rodríguez, T. (2017a). Comparaciones entre más de dos grupos: aportes para el cálculo del tamaño del efecto. Investigación en Educación Médica. Avance online. doi: 10.1016/j.riem.2017.04.003

Caycho-Rodríguez, T. (2017b). Tamaño del efecto e intervalos de confianza para correlaciones: aportes a Montes Hidalgo y Tomás-Sábado. Enfermería Clínica, 27(5), 331-332. doi: 10.1016/j.enfcli.2017.07.001

Caycho-Rodríguez, T., Ventura-León, J., Barboza-Palomino, M., Reyes-Bossio, M., Arias, W. L., García, C. H., Cabrera-Orosco, I., Ayala, J., \& Morgado-Gallardo, K. (2018). Validez e invarianza factorial por sexo de una medida breve de satisfacción con la vida familiar en escolares de Lima (Perú). Universitas Psychologica. Aceptado para publicación.

Cheung, F., \& Lucas, R. E. (2014). Assessing the validity of single-item life satisfaction measures: Results from three large samples. Quality of Life Research, 23(10), 2809-2818. doi: 10.1007/s11136-014-0726-4

Cohen, J. (1992). A power primer. Psychological Bulletin, 112(1), 155-159.

Conger, R. D., Conger, K. J., \& Martin, M. J. (2010). Socioeconomic status, family 
Evidencias preliminares de validez de contenido, validez convergente y confiabilidad del Item Único de Satisfacción con la vida familiar (IUSWFL) en adolescentes limeños

processes, and individual development. Journal of Marriage and Family, 72(3), 685-704. doi: 10.1111/j.1741-3737.2010.00725.x

Diener, E., \& Ryan, K. (2009). Subjective well-being: A general overview. South African Journal of Psychology, 39(4), 391-406. doi: 10.1177/008124630903900402

Dominguez-Lara, S. A. (2017). Ítem único de ansiedad ante exámenes: evidencias de validez convergente e incremental en estudiantes universitarios. Educación Médica. En prensa. doi: 10.1016/j.edumed.2017.04.004

Dominguez-Lara, S. A., \& Merino-Soto, C. (2015). ¿Por qué es importante reportar los intervalos de confianza del coeficiente alfa de Cronbach? Revista Latinoamericana de Ciencias Sociales, Niñez y Juventud, 13(2), 1326-1328.

Dominguez-Lara, S. A., \& Merino-Soto, C. (2017). Fiabilidad por consistencia interna de medidas de un solo ítem. Actas Urológicas Españolas, 41(3), 213. doi: 10.1016/j.acuro.2016.04.003

Easterlin, R. A. (2006). Life cycle happiness and its sources: Intersections of psychology, economics, and demography. Journal of Economic Psychology, 27(4), 463-482. doi: 10.1016/j.joep.2006.05.002

Feldt, L. S., Woodruff, D. J., \& Salih, F. A. (1987). Statistical inference for coefficient alpha. Applied Psychological Measurement, 11(1), 93-103. doi: 10.1177/014662168701100107

Ferguson, C. J. (2009). An effect size primer: A guide for clinicians and researchers. Professional Psychology: Research and Practice, 40(5), 532-538. doi: 10.1037/ aoo15808

Fernández-Arata, M., Merino-Soto, C., \& Dominguez-Lara, S. A. (2017). Ítem único en la medición del Burnout: resultados preliminares. Revista Cubana de Enfermería, 33(1). Recuperado de http://www.revenfermeria.sld.cu/index.php/ enf/article/view/1402

Frasquilho, D., Matos, M., Neville, F., Gaspar, T., \& Almeida, J. (2016). Parental Unemployment and Youth Life Satisfaction: The Moderating Roles of Satisfaction with Family Life. Journal of Child E Family Studies, 25(11), 3214-3219. doi:10.1007/s10826-016-048o-z

Fuchs, C., \& Diamantopoulos, A. (2009). Using single-item measures for construct measurement in management research: Conceptual issues and application guidelines. Die Betriebswirtschaft, 69(2), 195-210.

Gilman, R. (2001). The relationship between life satisfaction, social interest, and frequency of extracurricular activities among adolescent students. Journal of Youth and Adolescence, 30(6), 749-767. doi: 10.1023/A:1012285729701

Gilman, R., \& Huebner, S. (2003). A review of life satisfaction research with children and adolescents. School Psychology Quarterly, 18(2), 192-205. doi: 10.1521/ scpq.18.2.192.21858 
Tomás Caycho, Marilyn J. Cuellar, Renzo Carranza, Isabel Cabrera, Valeria Franco, Jennifer Casariego, Melanie Mendives, Lisseth Rojas \& Fiorella A. Torrejón

González-Serrano, G., Huéscar Hernández, E., \& Moreno-Murcia, J. A. (2013). Satisfacción con la vida y ejercicio físico. Motricidad. European Journal of Human Movement, 30, 131-151.

Huebner, E. S. (2004). Research on assessment of life satisfaction of children and adolescents. Social Indicators Research, 66(1-2), 3-33. doi: 10.1007/978-1-40202312-5_2

Huebner, E. S., Suldo, S. M., \& Gilman, R. (2006). Life Satisfaction. En G. G. Bear \& K. M. Minke (Eds.), Children's needs III: Development, prevention, and intervention (pp. 357-368). Washington, D. C.: National Association of School Psychologists.

Huebner, E. S., Suldo, S. M., Smith, L. C., \& McKnight, C. G. (2004). Life satisfaction in children and youth: Empirical foundations and implications for school psychologists. Psychology in the Schools, 41(1), 81-93. doi: 10.1002/pits.10140

Joo, S. H., \& Grable, J. E. (2004). An exploratory framework of the determinants of financial satisfaction. Journal of Family and Economic Issues, 25(1), 25-50. doi: 10.1023/B:JEEI.ooooo16722.37994.9f

Konrath, S., Meier, B. P., \& Bushman, B. J. (2018). Development and validation of the single item trait empathy scale (SITES). Journal of Research in Personality, 73, 111-122. doi: 10.1016/j.jrp.2017.11.009

Lautenschlager, G. J., \& Meade, A. W. (2008). AlphaTest: A Windows Program for Tests of Hypotheses About Coefficient Alpha. Applied Psychological Measurement, 32(6), 502-503. doi: 10.1177/0146621607312307

Lenzner, T., Kaczmirek, L., \& Lenzner, A. (2010). Cognitive burden of survey questions and response times: A psycholinguistic experiment. Applied Cognitive Psychology, 24(7), 1003-1020. doi: 10.1002/acp.1602

Lewis, A. D., Huebner, E., Malone, P. S., \& Valois, R. F. (2011). Life satisfaction and student engagement in adolescents. Journal of Youth and Adolescence, 40(3), 249-262. doi: 10.1007/s10964-010-9517-6

Loo, R. (2002). A caveat on using single-item versus multiple-item scales. Journal of Managerial Psychology, 17(1), 68-75. doi: 10.1108/o2683940210415933

Loo, R., \& Kelts, P. (1998). A caveat on using single-item measures. Employee Assistance Quarterly, 14(2), 75-8o. doi: 10.1300/Jo22v14no2_o6

Luna, A. C. A., Laca, F. A., \& Mejía, J. C. (2011). Bienestar subjetivo y satisfacción con la vida de familia en adolescentes mexicanos de bachillerato. Psicología Iberoamericana, 19(2), 17-26.

Merino, C., Navarro, J., \& García, W. (2014). Revisión de la consistencia interna del Inventario de Inteligencia Emocional de Bar-On, EQ-I: YV. Revista Peruana de Psicología y Trabajo Social, 3(1), 141-154. 
Evidencias preliminares de validez de contenido, validez convergente y confiabilidad del Item Único de Satisfacción con la vida familiar (IUSWFL) en adolescentes limeños

Moss, E., \& Willoughby, B. J. (2016). Associations between beliefs about marriage and life satisfaction: The moderating role of relationship status and gender. Journal of Family Studies. doi:10.108o/13229400.2016.1187658

Onwezen, M. C., Reinders, M. J., Verain, M. C. D., \& Snoek, H. M. (2019). The development of a single-item Food Choice Questionnaire. Food Quality and Preference, 71, 34-45. doi: 10.1016/j.foodqual.2018.05.005

Penfield, R. D., \& Giacobbi, P. R. (2004). Applying a score confidence interval to Aiken's item content-relevance index. Measurement in Physical Education and Exercise Science, 8(4), 213-225. doi: 10.1207/s15327841mpeeo804_3

Plagnol, A. C. (2011). Financial satisfaction over the life course: The influence of assets and liabilities. Journal of Economic Psychology, 32(1), 45-64. doi: 10.1016/j. joep.2010.10.006

Postmes, T., Haslam, S. A., \& Jans, L. (2013). A single-item measure of social identification: Reliability, validity, and utility. British Journal of Social Psychology, 52(4), 597-617. doi: 10.1111/bjso.12006

Proctor, C., Linley, P., \& Maltby, J. (2010). Very happy youths: Benefits of very high life satisfaction among adolescents. Social Indicators Research, 98(3), 519-532. doi: $10.1007 /$ s11205-009-9562-2

Serrano, M., \& Flores, M. (2004). Relaciones con los padres y apoyo social en adolescentes. Revista de Psicología de la Universidad Autónoma del Estado de México, 15-16, 87-101.

Shek, D., \& Li, X. (2016). Perceived School Performance, Life Satisfaction, and Hopelessness: A 4-Year Longitudinal Study of Adolescents in Hong Kong. Social Indicators Research, 126(2), 921-934. doi:10.1007/s11205-015-0904-y

Sloan, J. A., Aaronson, N., Cappelleri, J. C., Fairclough, D. L., Varricchio, C., \& Clinical Significance Consensus Meeting Group. (2002, May). Assessing the clinical significance of single items relative to summated scores. Mayo Clinic Proceedings, 77(5), 479-487. doi: 10.4065/77.5.479

Smilkstein, G. (1978). The family APGAR: A proposal for a family function test and its uses by physicians. The Journal of Family Practice, 6, 1231-1239.

Suldo, S. M., \& Shaffer, E. J. (2008). Looking beyond psychopathology: The dualfactor model of mental health in youth. School Psychology Review, 37(1), 52-68.

Suldo, S. M., Huebner, E. S., Friedrich, A. A., \& Gilman, R. (2009). Life satisfaction. En R. Gilman, E. S. Huebner, \& M. Furlong (Eds.), Handbook of Positive Psychology in the Schools (pp. 27-35). New York: Routledge.

Tașdelen-Karçkay, A. (2016). Family Life Satisfaction Scale - Turkish Version: Psychometric Evaluation. Social Behavior and Personality, 44(4), 631-640. doi: $10.2224 / \mathrm{sbp} .2016 .44 .4 .631$ 
Tomás Caycho, Marilyn J. Cuellar, Renzo Carranza, Isabel Cabrera, Valeria Franco, Jennifer Casariego, Melanie Mendives, Lisseth Rojas \& Fiorella A. Torrejón

Totan, T. T., Özer, A., \& Özmen, O. (2017). The role of hope, life satisfaction, and motivation in bullying among adolescents. International Online Journal of Educational Sciences, 9(2), 391-400. doi:10.15345/iojes.2017.02.008

Tsou, M. W., \& Liu, J. T. (2001). Happiness and domain satisfaction in Taiwan. Journal of Happiness Studies, 2(3), 269-288. doi: 10.1023/A:1011816429264

Valois, R. F., Zullig, K. J., Huebner, E. S., \& Drane, J. W. (2001). Relationship between life satisfaction and violent behaviors among adolescents. American Journal of Health Behavior, 25(4), 353-366. doi: 10.5993/AJHB.25.4.1

Van Hooff, M. L., Geurts, S. A., Kompier, M. A., \& Taris, T. W. (2007). "How fatigued do you currently feel?" Convergent and discriminant validity of a single-item fatigue measure. Journal of Occupational Health, 49(3), 224-234. doi: 10.1539/ joh.49.224

Van Praag, B. M., Van Praag, B., \& Ferrer-i-Carbonell, A. (2004). Happiness quantified: A satisfaction calculus approach. New York: Oxford University Press.

Ventura-León, J. L., \& Caycho, T. (2017). Q de Cohen: Comparación de Correlaciones entre Muestras Independientes en base a Urzúa et al. Revista Médica de Chile, 145(3), 411-412.

Wanous, J.P., \& Hudy, M.J. (2001). Single-item reliability:Areplicationand extension. Organizational Research Methods, 4(4), 361-375. doi: 10.1177/109442810144003

Wanous, J. P., Reichers, A. E., \& Hudy, M. J. (1997). Overall job satisfaction: how good are single-item measures? Journal of Applied Psychology, 82(2), 247-252.

West, C. P., Dyrbye, L. N., Satele, D. V., Sloan, J. A., \& Shanafelt, T. D. (2012). Concurrent validity of single-item measures of emotional exhaustion and depersonalization in burnout assessment. Journal of General Internal Medicine, 27(11), 1445-1452. doi: 10.1007/s116o6-012-2015-7

Yamamura, E. (2014). Smokers' sexual behavior and their satisfaction with family life. Social Indicators Research, 118(3), 1229-1247. doi: 10.1007/s11205-013-0466-9

Zabriskie, R. B., \& Ward, P. J. (2013). Satisfaction with family life scale. Marriage and Family Review, 49(5), 446-463. doi: 10.108o/01494929.2013.768321 